\title{
Micro-RNA expression in the urinary sediment of patients with chronic kidney diseases
}

\author{
Cheuk-Chun Szeto ${ }^{\mathrm{a}, *}$, Kwan Bonnie Ching-Ha ${ }^{\mathrm{a}}$, Lai Ka-Bik ${ }^{\mathrm{a}}$, Lai Fernand Mac-Moune ${ }^{\mathrm{b}}$, \\ Choi Paul Cheung-Lung ${ }^{\mathrm{b}}$, Wang Gang ${ }^{\mathrm{a}}$, Chow Kai-Ming and ${ }^{\mathrm{a}}$ Li Philip Kam-Tao ${ }^{\mathrm{a}}$ \\ ${ }^{a}$ Department of Medicine \& Therapeutics, Prince of Wales Hospital, The Chinese University of Hong Kong, Shatin, \\ Hong Kong, China \\ ${ }^{\mathrm{b}}$ Department of Anatomical \& Cellular Pathology, Prince of Wales Hospital, The Chinese University of Hong \\ Kong, Shatin, Hong Kong, China
}

\begin{abstract}
Background: Evidence indicates that microRNAs (miRNA) play a role in the pathogenesis of chronic kidney diseases (CKD). We explored the possibility of using urinary miRNA as non-invasive biomarkers for CKD.

Methods: We quantified miRNA expression in urinary sediment of 56 CKD patients who underwent kidney biopsy. Patients were followed for $16.2 \pm 15.5$ months.

Results: Patients with diabetic glomerulosclerosis had lower urinary miR-15 expression, while those with IgA nephropathy had higher urinary miR-17 expression, than other diagnosis groups. Baseline proteinuria had significant inverse correlation with urinary expression of miR-15, miR-192, and miR-216a; baseline renal function correlated with urinary expression of miR-15, miR-17, miR-192, and miR-217. The rate of renal function decline correlated with urinary expression of miR-21 $(r=0.301$, $p=0.026)$ and miR-216a $(r=0.515, p<0.0001)$. Patients with a high urinary expression of miR-21 and miR-216a had better dialysis-free survival than those with low expression (log rank test, $p=0.005$ and $p=0.003$, respectively).

Conclusions: Urinary miR-21 and miR-216a expression correlated with the rate of renal function decline and risk of progression to dialysis-dependent renal failure. Our results suggest that urinary miRNA profiling has the potential of further development as biomarkers of CKD.
\end{abstract}

Keywords: Proteinuria, glomerulonephritis, biomarker

\section{Introduction}

Chronic kidney disease (CKD) is a debilitating and costly medical condition. The clinical course is characterized by persistent proteinuria after an initial insult to the kidney, followed by progressive decline in renal function [1]. Reliable markers are much needed for the clinical management of CKD. Proteinuria and the severity of tubulo-interstitial scarring in kidney biopsy are the most commonly used prognostic indicators in CKD patients [1-3]. However, neither of them is an entirely satisfactory marker of CKD.

*Correspondence: Dr. C.-C. Szeto, Department of Medicine \& Therapeutics, Prince of Wales Hospital, The Chinese University of Hong Kong, Shatin, NT, Hong Kong, China. Tel.: +852 2632 3126; Fax: +852 2637 3852; E-mail: ccszeto@cuhk.edu.hk.
Micro-RNAs (miRNAs) are short noncoding RNA molecules that inhibit gene expression through incomplete base pairing with the 3'-untranslated region of target mRNAs [4,5]. Evidence indicates that miRNAs play a role in the pathogenesis of many human diseases [6,7]. Recently, miRNA control has also been found to have a critical regulatory role in epithelialmesenchymal transition (EMT) [8], which may play an important role in the progressive kidney damage in CKD [9]. Recently, miRNA level could be measured in serum and other body fluids [10]. In urine, miRNA could be quantified in both the urinary sediment as well as the supernatant after centrifugation [10].

The role of urinary miRNA as biomarkers of kidney disease is under active research. There are several advantages of quantifying miRNA in urine as biomarkers. First, although the measurement of cytokines and 
growth factors in urine would seem logical means to assess the severity of kidney damage and provide prognostic information, measurement of urinary cytokine protein may not be clinically relevant because most of the cytokines exert paracrine effects and are not excreted in the urine, and the amount of cytokine protein found in urine may not correlate with the local concentration in tubulointerstitium [11]. Furthermore, there is a limited number of human miRNAs, with each individual miRNA modulating protein output from hundreds of target genes [12]. In addition, miRNA is relatively stable and resistant to degradation, making it an ideal substance to be tested for large scale clinical use or research with archive specimens.

We have previously reported that urinary expression of miR-200a, miR-200b and miR-429 were downregulated in patients with IgA nephropathy, and the degree of reduction correlated with disease severity and rate of progression [13], while patients with active lupus nephritis had lower urinary expression of miR200a, miR-200c, miR-141, miR-429 and miR-192 than healthy controls [14]. In the present study, we explore the possibility of using urinary miRNA as non-invasive biomarkers for CKD.

\section{Patients and methods}

\subsection{Case selection}

We studied 56 consecutive patients who underwent kidney biopsy between January 2009 and June 2010 in the Prince of Wales Hospital, Hong Kong. Patients with acute renal failure or active glomerulonephritis were excluded. The study was approved by the Clinical Research Ethical Committee of the Chinese University of Hong Kong, all patients provided fully informed consent. A whole-stream early morning urine specimen was collected by each patient on the biopsy day for urinary miRNA expression study. Clinical data including serum creatinine and 24 hours urine protein were recorded. Glomerular filtration rate (GFR) was estimated by a standard equation [15].

\subsection{Quantification of urinary miRNA}

The methods of urinary miRNA extraction and quantification have been described in our previous studies $[13,14]$. Briefly, urine specimen was collected and sent to laboratory for processing immediately or stored in $4^{\circ} \mathrm{C}$ overnight. Urine sample was centrifuged at
$3000 \mathrm{~g}$ for 30 minutes and at $13000 \mathrm{~g}$ for 5 minutes at $4{ }^{\circ} \mathrm{C}$. Supernatant was discarded and the urinary cell pellet was lysed by RNA lysis buffer (Qiagen Inc, Ontario, Canada). Specimens were then stored at $-80^{\circ} \mathrm{C}$ until use.

MirVana $^{\mathrm{TM}}$ miRNA isolation kit (Ambion, Inc. Austin, TX, USA) was used for the extraction of total RNA from urinary sediment according to the manufacturer's protocol. We confirmed the purity of urinary RNA by the relative absorbance at $260 / 280 \mathrm{~nm}$ ratio using a spectrometer (Hitachi, Japan). Our previous data have shown the integrity of RNA isolated from urinary sediment by this method is adequate for real time quantitative polymerase chain reaction (RT-QPCR).

TaqMan ${ }^{\circledR}$ miRNA reverse transcription Kit (Applied Biosystems, Foster City, CA, USA) and High Capacity cDNA Reverse Transcription Kit (Applied Biosystems, Foster City, CA, USA) were used for reverse transcription. For miRNA, $5 \mu$ l total RNA was mixed with $3 \mu \mathrm{l}$ specific primers, $0.15 \mu \mathrm{l} 10 \mathrm{mM}$ dNTPs (with dTTP), $1.5 \mu \mathrm{l} 10 \mathrm{x}$ reverse transcription buffer, $1 \mu \mathrm{l}$ (50U) MultiScribe ${ }^{\mathrm{TM}}$ Reverse Transcriptase $0.19 \mu \mathrm{l}$ RNase inhibitor $(20 \mathrm{U} / \mu \mathrm{l})$ and made up to $15 \mu \mathrm{l}$ with $\mathrm{H}_{2} \mathrm{O}$. Reverse transcription was performed at $16^{\circ} \mathrm{C}$ for 30 minutes, $42^{\circ} \mathrm{C}$ for 30 minutes and $85^{\circ} \mathrm{C}$ for 5 minutes. For messenger RNA, $10 \mu 1$ total RNA was mixed with $2 \mu \mathrm{l}$ specific primers, $0.8 \mu \mathrm{l} 10 \mathrm{mM}$ dNTPs (with dTTP), $2 \mu \mathrm{l}$ 10x reverse transcription buffer, $1 \mu \mathrm{l}$ (50U) MultiScribe $^{\mathrm{TM}}$ Reverse Transcriptase $1 \mu \mathrm{l}$ RNase inhibitor $(20 \mathrm{U} / \mu \mathrm{l})$ and made up to $20 \mu \mathrm{l}$ with $\mathrm{H}_{2} \mathrm{O}$. Reverse transcription was performed at $25^{\circ} \mathrm{C}$ for $10 \mathrm{~min}$ utes, $37^{\circ} \mathrm{C}$ for 120 minutes and $85^{\circ} \mathrm{C}$ for 5 minutes. The resulting cDNA was stored in $-80^{\circ} \mathrm{C}$ until use.

Urinary expression of miR-15, miR-17, miR-21, miR-30, miR-192, miR-216a, miR-217, and miR-377 were quantified by RT-QPCR using the ABI Prism 7900 Sequence Detection System (Applied Biosystems, Foster City, CA, USA). These targets were chosen because of their reported involvement in renal fibrosis and progression of CKD [16,17]. Commercially available Taqman primers and probes, including 2 unlabeled PCR primers and 1 FAM $^{\mathrm{TM}}$ dye-labeled TaqMan ${ }^{\circledR}$ MGB probe were used for all the targets (all from Applied Biosystems). Each sample was run in triplicate. RTQPCR were performed at $50^{\circ} \mathrm{C}$ for 2 minutes, $95^{\circ} \mathrm{C}$ for 10 minutes, followed by 40 cycles at $95^{\circ} \mathrm{C}$ for 15 seconds and $60^{\circ} \mathrm{C}$ for 1 minute. Small RNA U6 (Applied Biosystems) was used as house-keeping genes to normalize the microRNA expression $[18,19]$. Results were analyzed with Sequence Detection Software version 2.0 (Applied Biosystems). In order to calculate the 
Table 1

Demographic and clinical data of the study patients

\begin{tabular}{|c|c|c|c|}
\hline Group & $\operatorname{IgAN}$ & DGS & HTN \\
\hline No. of patients & 17 & 17 & 22 \\
\hline $\operatorname{Sex}(M: F)$ & $5: 12$ & $10: 7$ & $12: 10$ \\
\hline Age (years) & $47.8 \pm 12.6$ & $52.5 \pm 12.6$ & $56.3 \pm 14.0$ \\
\hline \multicolumn{4}{|l|}{ Renal function } \\
\hline Proteinuria $(\mathrm{g} / \mathrm{day})^{*}$ & $2.9 \pm 2.1$ & $6.6 \pm 3.1$ & $3.0 \pm 3.0$ \\
\hline Serum creatinine $(\mu \mathrm{mol} / \mathrm{l})$ & $235.6 \pm 193.9$ & $292.9 \pm 160.3$ & $270.4 \pm 223.7$ \\
\hline $\operatorname{GFR}\left(\mathrm{ml} / \mathrm{min} / 1.73 \mathrm{~m}^{2}\right)$ & $46.7 \pm 41.1$ & $28.2 \pm 21.5$ & $34.5 \pm 22.1$ \\
\hline \multicolumn{4}{|l|}{ Histological scarring } \\
\hline Glomerulosclerosis (\%) & $39.0 \pm 31.3$ & $28.4 \pm 16.8$ & $45.1 \pm 31.4$ \\
\hline Tubulointerstitial fibrosis (\%) & $36.3 \pm 27.2$ & $54.1 \pm 14.1$ & $39.9 \pm 27.6$ \\
\hline GFR decline $\left(\mathrm{ml} / \mathrm{min} / 1.73 \mathrm{~m}^{2} \text { per year }\right)^{* *}$ & $-10.2 \pm 16.4$ & $-14.1 \pm 11.5$ & $-3.6 \pm 6.3$ \\
\hline
\end{tabular}

IgAN, IgA nephropathy; DGS, diabetic glomerulosclerosis; HTN, hypertensive nephrosclerosis; GFR, glomerular filtration rate. ${ }^{*} p<0.0001$ by one-way ANOVA; ${ }^{* *} p=0.005$ by Kruskal-Wallis test.

differences of expression level for each target among samples, the $\Delta \Delta \mathrm{C}_{T}$ method for relative quantitation was used.

\subsection{Assessment of renal scarring}

Analysis of renal fibrosis was determined on $4 \mu \mathrm{m}$ paraffin-embedded sections stained by Periodic Acid Schiff (PAS) or Jones silver stain. The severity of renal fibrosis was scored subjectively by an experienced pathologist who was blinded to the results of molecular studies. The severity of glomerulosclerosis was represented by the percentage of sclerotic glomeruli in total glomeruli obtained from biopsy. For tubulointerstitial scarring, ten microscopic fields were viewed at magnification of $200 \times$ and scored subjectively from 0 to $100 \%$ for each patients. The severity of tubulointerstitial scarring was represented by the mean of ten scores.

\subsection{Clinical management}

After renal biopsy, all patients were followed every 2 months for at least 12 months Renal function and proteinuria levels were assessed at least every 4 months. Disease progression was measured by the rate of GFR decline, which was calculated by the least-square regression method [20]. Treatment for individual patient was determined by responsible physician and not affected by this study. All physicians were blinded from the results of RNA expression.

\subsection{Statistical analysis}

Statistical analysis was performed by SPSS for Windows software version 15.0 (SPSS Inc., Chicago, IL).
All the results were presented in mean \pm SD for data normally distributed and median (lower and upper quartiles) for the others. Since data of gene expression levels were highly skewed, either log transformation or nonparametric statistical methods were used. When no detectable level of a transcript was found (defined as no detectable level after 40 cycles of RT-QPCR) and there was zero value, a value equal to half of the minimum observed gene expression level was assigned.

We used Kruskal Wallis test or Mann-Whitney U test to compare gene expression levels between groups and Spearman's rank-order correlations to test associations between gene expression levels and clinical parameters. For the analysis of dialysis-free survival, Kaplan-Meier survival curves were constructed. Gene expression of each target was classified into high and low groups according to the median level of expression and then compared by the log rank test. A P value of below 0.05 was considered statistically significant. All probabilities were two-tailed.

\section{Results}

We studied 56 CKD patients. The underlying histological diagnosis were IgA nephropathy (17 cases), diabetic nephrosclerosis (17 cases), and hypertensive nephrosclerosis (22 cases). The baseline demographic and clinical data of the patients are summarized in Table 1. None of the patients with IgA nephropathy had necrotizing or crescentic lesion in renal biopsy. In short, most of the baseline clinical and biochemical parameters were highly comparable between the diagnosis groups, except patients with diabetic glomerulosclerosis had more proteinuria $(p<0.0001)$ and marginally more tubulointerstitial scarring $(p=0.09)$ than the other groups. 
Table 2

Internal correlations between urinary expression of miRNA targets

\begin{tabular}{|c|c|c|c|c|c|c|c|}
\hline & miR-17 & miR-21 & miR-30 & miR-192 & miR-216a & miR-217 & miR-377 \\
\hline miR-15 & $\begin{array}{l}r=0.619, \\
p<0.0001\end{array}$ & $\begin{array}{l}r=0.776 \\
p<0.0001\end{array}$ & $\begin{array}{l}r=0.364 \\
p=0.006\end{array}$ & $\begin{array}{l}r=0.541 \\
p<0.0001\end{array}$ & $\begin{array}{l}r=0.319 \\
p=0.017\end{array}$ & $\begin{array}{l}r=0.424 \\
p=0.001\end{array}$ & $\begin{array}{l}r=-0.413, \\
p=0.002\end{array}$ \\
\hline miR-17 & & $\begin{array}{l}r=0.500 \\
p=0.0001\end{array}$ & $\begin{array}{l}r=0.560 \\
p<0.0001\end{array}$ & $\begin{array}{l}r=0.607 \\
p<0.0001\end{array}$ & $\begin{array}{l}r=0.271, \\
p=0.04\end{array}$ & $\begin{array}{l}r=0.353 \\
p=0.008\end{array}$ & $\begin{array}{l}r=-0.350 \\
p=0.008\end{array}$ \\
\hline miR-21 & & & $\begin{array}{l}p=0.636 \\
p<0.0001\end{array}$ & $\begin{array}{l}r=0.758 \\
p<0.0001\end{array}$ & $\begin{array}{l}r=0.423 \\
p=0.001\end{array}$ & $\begin{array}{l}r=0.595 \\
p<0.0001\end{array}$ & $\begin{array}{l}r=-0.301, \\
p=0.024\end{array}$ \\
\hline miR-30 & & & & $\begin{array}{l}r=0.903 \\
p<0.0001\end{array}$ & $\begin{array}{l}r=0.600 \\
p<0.0001\end{array}$ & $\begin{array}{l}r=0.629 \\
p<0.0001\end{array}$ & $\begin{array}{l}r=-0.126 \\
p=0.4\end{array}$ \\
\hline miR-192 & & & & & $\begin{array}{l}r=0.630 \\
p<0.0001\end{array}$ & $\begin{array}{l}r=0.749 \\
p<0.0001\end{array}$ & $\begin{array}{l}r=-0.166, \\
p=0.2\end{array}$ \\
\hline miR-216a & & & & & & $\begin{array}{l}r=0.394 \\
p=0.003\end{array}$ & $\begin{array}{l}r=0.113 \\
p=0.4\end{array}$ \\
\hline miR-217 & & & & & & & $\begin{array}{l}r=-0.190, \\
p=0.16\end{array}$ \\
\hline
\end{tabular}
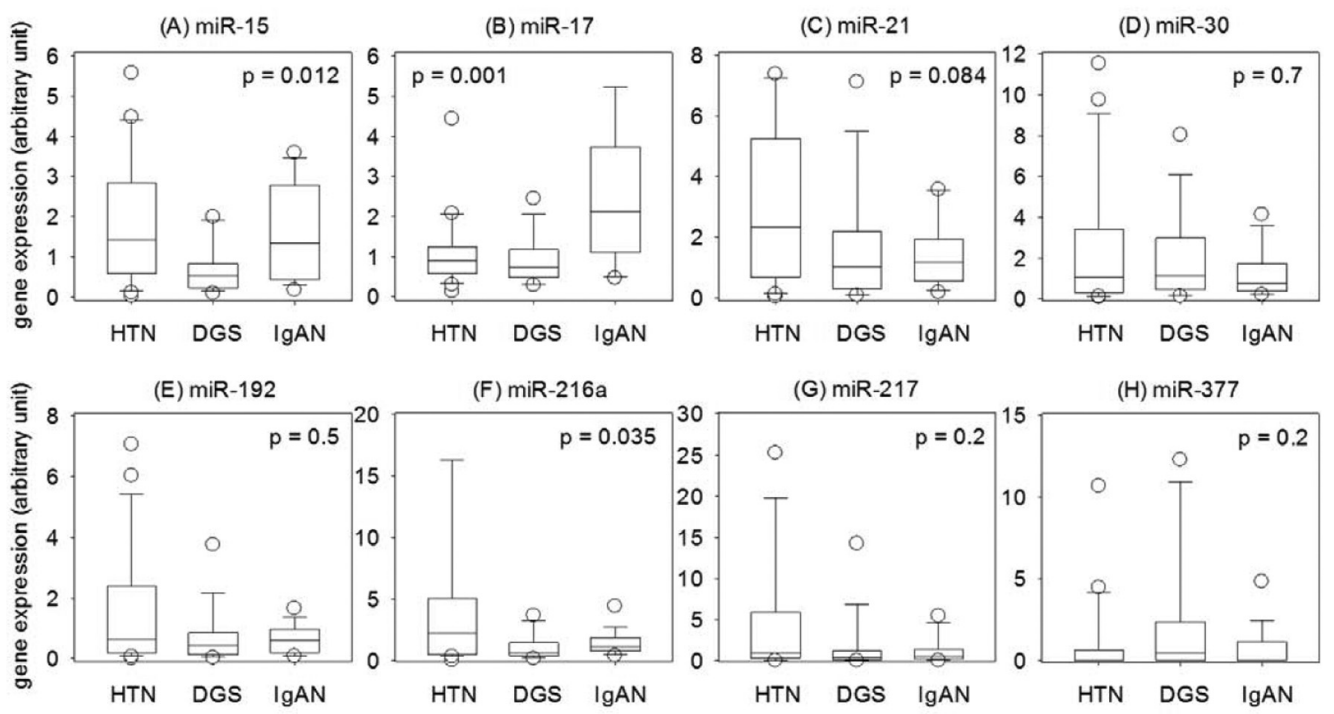

Fig. 1. Comparison of miRNA expression in urinary sediment between diagnosis groups: (A) miR-15; (B) miR-17; (C) miR-21; (D) miR-30; (E) miR-192; (F) miR-216a; (G) miR-217; and (H) miR-377. Whisker-box plot, with the boxes indicate median, 25 and 75 percentile; whisker caps indicate 5 and 95 percentile; circles indicate outliers. Data are compared by Kruskal Wallis test. (Key: HTN, hypertensive nephrosclerosis; DGS, diabetic glomerulosclerosis; IgAN, IgA nephropathy.)

\subsection{Difference between groups}

There was a close internal correlation between most of the urinary expressions of the miRNA targets except miR-377 (Table 2). Urinary expression of individual miRNA targets are compared between diagnosis groups and summarized Fig. 1. Briefly, we found that patients with diabetic glomerulosclerosis had lower urinary miR-15 expression than the other groups, when patients with IgA nephropathy had higher urinary miR-17 expression than other groups. Patients with hypertensive nephrosclerosis tended to have higher urinary expression of miR-216a and miR-21, although the latter did not reach statistical significance.

\subsection{Relation with baseline characteristics}

The relation between urinary expressions of miRNA targets and proteinuria, baseline renal function, degree of glomerulosclerosis and tubulointerstitial fibrosis is summarized in Table 3. In essence, baseline proteinuria had significant inverse correlation with urinary expression of miR-15, miR-192, and miR-216a, while baseline renal function positively correlated with urinary expression of miR-15, miR-17, miR-192, and miR-217. The degree of tubulointerstitial fibrosis positively correlated with urinary expression of miR-377, and inversely with urinary miR-217, while no significant correlation was observed between the degree of 
Table 3

Relation between urinary expression of miRNA targets and baseline clinical and pathological parameters

\begin{tabular}{lcccc}
\hline & Proteinuria & Estimated GFR & Glomerulosclerosis & Tubulointerstitial fibrosis \\
\hline miR-15 & $r=-0.355, p=0.008$ & $r=0.277, p=0.04$ & $r=-0.032, p=0.8$ & $r=-0.256, p=0.06$ \\
miR-17 & $r=-0.228, p=0.1$ & $r=0.359, p=0.007$ & $r=-0.142, p=0.3$ & $r=-0.228, p=0.1$ \\
miR-21 & $r=-0.160, p=0.2$ & $r=0.163, p=0.2$ & $r=0.006, p=0.9$ & $r=-0.137, p=0.3$ \\
miR-30 & $r=-0.075, p=0.6$ & $r=0.226, p=0.09$ & $r=-0.046, p=0.7$ & $r=-0.137, p=0.3$ \\
miR-192 & $r=-0.284, p=0.04$ & $r=0.370, p=0.005$ & $r=-0.119, p=0.4$ & $r=-0.261, p=0.06$ \\
miR-216a & $r=-0.430, p=0.001$ & $r=0.131, p=0.3$ & $r=0.149, p=0.3$ & $r=-0.200, p=0.15$ \\
miR-217 & $r=-0.261, p=0.06$ & $r=0.398, p=0.002$ & $r=-0.010, p=0.9$ & $r=-0.354, p=0.009$ \\
miR-377 & $r=0.097, p=0.5$ & $r=-0.145, p=0.3$ & $r=0.020, p=0.9$ & $r=0.313, p=0.02$ \\
\hline
\end{tabular}

GFR, glomerular filtration rate.
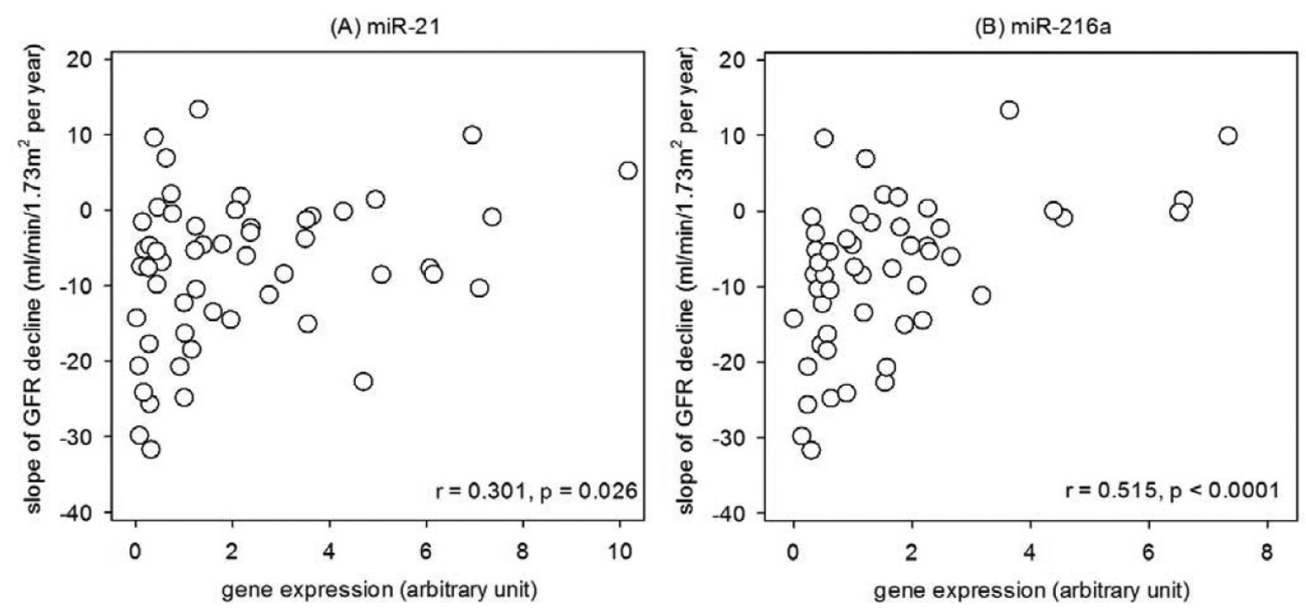

Fig. 2. Relation between the rate of glomerular filtration rate (GFR) decline and urinary expression of (A) miR-21; and (B) miR-216a. Data are compared by Spearman's rank correlation coefficient.

glomerulosclerosis and the urinary expression of any miRNA target examined in this study.

\subsection{Relation with renal function decline}

Patients were followed for an average of $16.2 \pm$ 15.5 months. All patients received angiotensin converting enzyme inhibitor or angiotensin receptor blocker therapy; none of the patient with IgA nephropathy received corticosteroid or immunosuppressive treatment. The average rate of GFR decline was $-8.8 \pm$ $12.4 \mathrm{ml} / \mathrm{min} / 1.73 \mathrm{~m}^{2}$ per year. Patients with hypertensive nephrosclerosis had a significantly slower rate of GFR decline as compared to other diagnosis groups (see Table 1). When all patients were pooled and analyzed, the rate of GFR decline positively correlated with the urinary expression of miR-21 $(r=0.301, p=$ 0.026) and miR-216a ( $r=0.515, p<0.0001)$ (Fig. 2). When the three diagnosis groups were analyzed separately, urinary miR-216a expression correlated with the rate of GFR decline in patients with hypertensive nephrosclerosis $(r=0.588, p=0.005)$ and diabetic glomerulosclerosis ( $r=0.605, p=0.010)$, but not $\operatorname{IgA}$ nephropathy ( $r=0.042, p=0.9)$. Urinary miR-21 expression did not correlate with the rate of GFR decline for any diagnosis group.

During follow up, 18 patients (5 patients with IgA nephropathy, 8 with diabetic glomerulosclerosis; and 5 with hypertensive nephrosclerosis) progressed to end stage renal disease and were put on dialysis. KaplanMeier analysis showed that patients with a high urinary expression of miR-21 and miR-216a had better dialysis-free survival than those with low expression (log rank test, $p=0.005$ and $p=0.003$, respectively) (Fig. 3). Other miRNA targets tested in this experiment were not associated with dialysis-free survival (details not shown). Because the number of event was small, extensive multivariate survival analysis was not performed.

\section{Discussion}

In the present study, we found that urinary expression of miR-15, miR-17, and miR-216a were significantly 

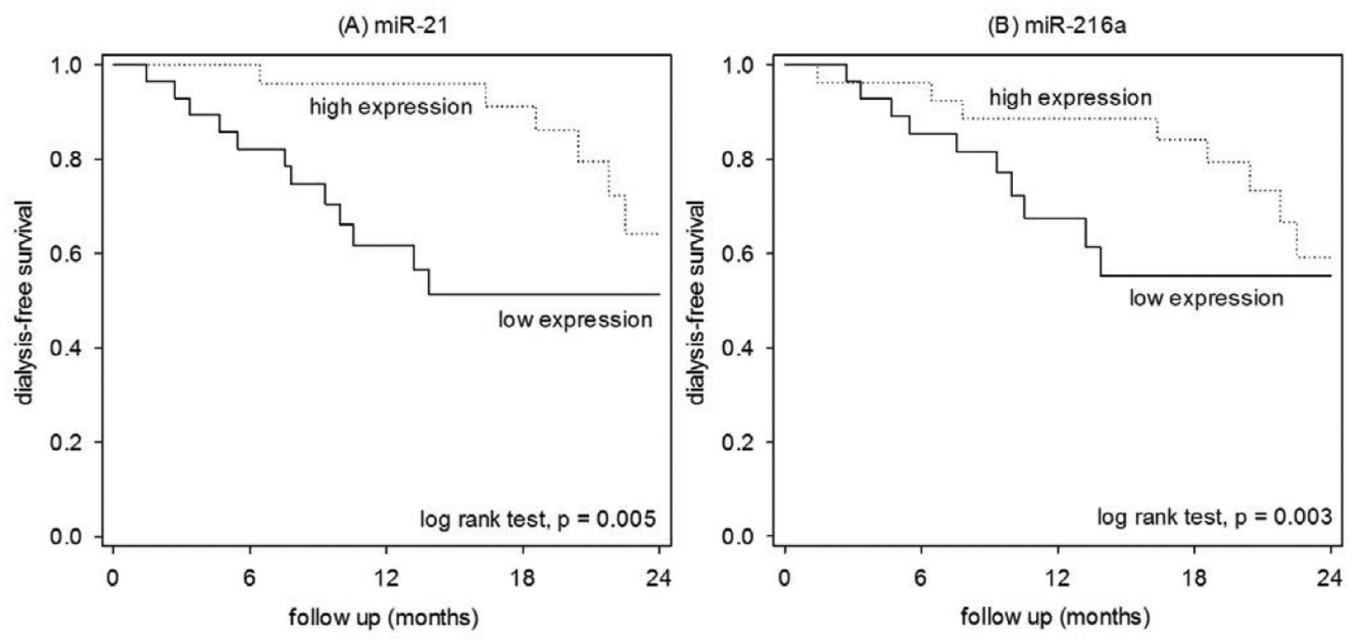

Fig. 3. Kaplan-Meier plot of dialysis-free survival with respect to the urinary expression of (A) miR-21; and (B) miR-216a. For either miRNA, patients were divided into two groups according to the median level of expression, which was 25 copies per 100,000 copies of the housekeeping gene. Data are compared by log rank test.

different between diagnosis groups. The expression of some miRNA targets in the urinary sediment correlated with proteinuria, renal function, and the degree of tubulointerstitial fibrosis. In addition, urinary miR-21 and miR-216a expression correlated with the rate of renal function decline as well as the risk of progression to dialysis-dependent renal failure. Our results suggest that urinary miRNA profiling has the potential of further development as biomarkers of chronic kidney diseases.

Our result is, in general, consistent with previous reports. For example, Kato et al. [21] showed that miR192 levels were enhanced significantly in glomeruli isolated from streptozotocin-injected diabetic mice as well as diabetic $\mathrm{db} / \mathrm{db}$ mice, and miR-192 contributes to the TGF-beta-induced collagen expression via inhibition of E-box repressors. The same group also found that miR-216a and miR-217 target phosphatase and tensin homologue (PTEN), which in turn regulates Akt activation and TGF-beta-induced fibrosis [22]. With the same animal models of diabetic nephropathy, Zhang et al. [23] showed that miR-21 expression was downregulated in response to early diabetic nephropathy, and over-expression of miR-21 inhibited proliferation of mesangial cells and decreased urine albumin excretion rate in diabetic $\mathrm{db} / \mathrm{db}$ mice. Wang et al. [24] reported that overexpression of miR-377 in diabetic nephropathy leads to increased fibronectin production via reduced expressions of p21-activated kinase and superoxide dismutase. Similarly, Lee et al. [25] found that miR-15 contributes to the cystogenesis of polycystic kidney disease, while, in a similar model, Sun et al. [26] showed that overexpression of miR-17 may promote cell proliferation via post-transcriptionally repression of target genes. Taken together, our results further support the hypothesis that the above-mentioned miRNA targets may contribute to the progression of renal failure in CKD.

In addition to shedding light on the pathogenic role of miRNA in progressive CKD, another important reason to explore miRNA expression in urinary sediment of CKD patient is biomarker development. In fact, there are several advantages of quantifying miRNA in urine as compared to the measurement of messenger RNA. First, there is a limited number of human miRNAs, with each individual miRNA modulating protein output from hundreds of target genes [12]. Second, miRNA is relatively stable and resistant to degradation, making it an ideal substance to be tested for large scale clinical use or research with archive specimens. Finally, effective means of in vivo introduction of miRNA is being developed [27], and any relevant miRNA species may have a therapeutic potential in the future.

A number of previous studies from our group had explored the role of urinary miRNA level as the biomarker for various forms of kidney diseases [13,14,28,29]. Notably, our previous study showed that urinary levels of miR-146a and miR-155 correlated with the levels of various inflammatory markers in IgA nephropathy [28] and lupus nephritis [29]. In the present study, we focused on the miRNA targets that are reported to be involved in the development and progression of renal 
fibrosis in CKD in general $[16,17]$, rather than those miRNA targets relevant for immune responses (such as miR-146a and miR-155) in specific inflammatory glomerulonephritis.

There are a few inadequacies of our study. First, we detected the expression levels of the studied miRNAs using urine sediment without determining the cellular sources for each of them. Although not directly proved, our previous experience suggests that urinary miRNAs in the present study are most likely from deciduous tubular epithelial cells and podocytes [28]. Previous studies, however, showed that miR-21, miR-192, miR216a, and miR-377 are largely expressed in mesangial cells, while miR-15 and mR-17 are probably expressed in tubular epithelial cells $[16,17]$. Future studies would be necessary to investigate miRNA expression level in specific renal cell type. To further delineate the role of individual miRNA species in the progression of CKD, it would also be necessary to study their intra-renal expression in renal biopsy specimens. Since we aimed to determine the relation between urinary miRNA level and CKD progression, we did not include a control group with normal kidney function for comparison. Recently study, however, showed that urinary miR-21 levels are elevated in patients with microalbuminuria and early CKD as compared to healthy schoolchildren [30].

Second, the scope of our present study is limited, both in terms of renal diagnosis and miRNA targets that were tested. Since we only studied three common renal diseases, our result may not be extrapolated to CKD in general. In addition, the overall sample size was small, so that we might not have sufficient statistical power for a proper multivariate analysis and determine the prognostic role of urinary miRNA expression. Notably, proteinuria is one of the best prognostic indictor of CKD progression. Our present study did not confirm that urinary miRNA quantification provides additional prognostic information after adjusting for the degree of proteinuria, and further validation study is necessary on this respect.

Also because of the small sample size of our study, therapeutic intervention of the recruited patients could have be heterogeneous. Nonetheless, we put the utmost effort to study a homogeneous population of CKD patients treated with standard therapy (blood pressure control, renin-angiotensin-axis blockade, and no immunosuppressive therapy). In this study, we chose specific miRNA targets, based on their reported involvement in renal fibrosis and progression of CKD [16,17]. Further study is also needed to screen for the expression of all possible miRNA species by a hypothesis-free technology (for example, microarray).

\section{Acknowledgement}

This study was supported in part by the CUHK research account 6901031. All authors declare no conflict of interest. The results presented in this paper have not been published previously in whole or part, except in abstract format.

\section{Conflict of interest}

All authors declare no conflict of interest. The results presented in this paper have not been published previously in whole or part, except in abstract format.

\section{References}

[1] Remuzzi G, Bertani T. Pathophysiology of progressive nephropathies. N Engl J Med 1998; 339: 1448-1456.

[2] Keane WF. Proteinuria: its clinical importance and role in progressive renal disease. Am J Kidney Dis 2000; 35 (Suppl 1): S97-105.

[3] Becker GJ, Hewitson TD. The role of tubulointerstitial injury in chronic renal failure. Curr Opin Nephrol Hypertens 2000; 9: 133-138.

[4] Meister G, Tuschl T. Mechanisms of gene silencing by doublestranded RNA. Nature 2004; 431: 343-349.

[5] Bartel DP. MicroRNAs: genomics, biogenesis, mechanism and function. Cell 2004; 116: 281-297.

[6] Bartels CL, Tsongalis GJ. MicroRNAs: Novel Biomarkers for Human Cancer. Clin Chem 2009; 55: 623-631.

[7] Xiao C, Rajewsky K. MicroRNA control in the immune system: basic principles. Cell 2009; 136: 26-36.

[8] Gregory PA, Bracken CP, Bert AG, Goodall GJ. MicroRNAs as regulators of epithelial-mesenchymal transition. Cell Cycle 2008; 7: 3112-3118.

[9] Neilson EG. Mechanisms of disease: Fibroblasts - a new look at an old problem. Nat Clin Pract Nephrol 2006; 2: 101-108.

[10] Gilad S, Meiri E, Yogev Y, Benjamin S, Lebanony D, Yerushalmi N, Benjamin H, Kushnir M, Cholakh H, Melamed N, Bentwich Z, Hod M, Goren Y, Chajut A. Serum microRNAs are promising novel biomarkers. PLoS ONE 2008; 3: e3148.

[11] Wenzel UO, Abboud HE. Chemokines and renal disease. Am J Kidney Dis 1995; 26: 982-994.

[12] Asirvatham AJ, Magner WJ, Tomasi TB. miRNA regulation of cytokine genes. Cytokine 2009; 45: 58-69.

[13] Wang G, Kwan BC, Lai FM, Chow KM, Li PK, Szeto CC. Expression of microRNAs in the urinary sediment of patients with IgA nephropathy. Dis Marker 2010; 28: 79-86.

[14] Wang G, Tam LS, Li EK, Kwan BC, Chow KM, Luk CC, Li PK, Szeto CC. Serum and urinary free microRNA level in patients with systemic lupus erythematosus. Lupus 2011; 20: 493-500.

[15] Levey AS, Bosch JP, Lewis JB, Greene T, Rogers N, Roth D: A more accurate method to estimate glomerular filtration rate from serum creatinine: a new prediction equation. Modification of Diet in Renal Disease Study Group. Ann Intern Med 1999, 130(6): 461-470. 
[16] Lorenzen JM, Haller H, Thum T. MicroRNAs as mediators and therapeutic targets in chronic kidney disease. Nat Rev Nephrol 2011; 7: 286-294.

[17] Li JY, Yong TY, Michael MZ, Gleadle JM. The role of microRNAs in kidney disease. Nephrology (Carlton) 2010; 15: 599-608.

[18] Bhandari S, Watson N, Long E, Sharpe S, Zhong W, Xu SZ, Atkin SL: Expression of somatostatin and somatostatin receptor subtypes 1-5 in human normal and diseased kidney. $\mathrm{J}$ Histochem Cytochem 2008, 56(8):733-743.

[19] AppliedBiosystems: Endogenous Controls for Real-Time Quantitation of miRNA Using TaqMan ${ }^{\circledR}$ MicroRNA Assays. Application Note TaqMan ${ }^{\circledR}$ MicroRNA Assays 2007

[20] Szeto CC, Leung CB, Wong TY, Chow KM, Wang AY, Lui SF, $\mathrm{Li}$ PK. Extrapolation of reciprocal creatinine plot is not reliable in predicting the onset of dialysis in patients with progressive renal insufficiency. J Intern Med 2003; 253: 335-342.

[21] Kato M, Zhang J, Wang M, Lanting L, Yuan H, Rossi JJ, Natarajan R. MicroRNA-192 in diabetic kidney glomeruli and its function in TGF-beta-induced collagen expression via inhibition of E-box repressors. Proc Natl Acad Sci USA 2007; 104: 3432-3437.

[22] Kato M, Putta S, Wang M, Yuan H, Lanting L, Nair I, Gunn A, Nakagawa Y, Shimano H, Todorov I, Rossi JJ, Natarajan R. TGF-beta activates Akt kinase through a microRNAdependent amplifying circuit targeting PTEN. Nat Cell Biol 2009; 11: 881-889.

[23] Zhang Z, Peng H, Chen J, Chen X, Han F, Xu X, He X, Yan N. MicroRNA-21 protects from mesangial cell proliferation induced by diabetic nephropathy in $\mathrm{db} / \mathrm{db}$ mice. FEBS Lett 2009; 583: 2009-2014.
[24] Wang Q, Wang Y, Minto AW, Wang J, Shi Q, Li X, Quigg RJ. MicroRNA-377 is up-regulated and can lead to increased fibronectin production in diabetic nephropathy. FASEB J 2008; 22: 4126-4135.

[25] Lee SO, Masyuk T, Splinter P, Banales JM, Masyuk A, Stroope A, Larusso N. MicroRNA15a modulates expression of the cell-cycle regulator $\mathrm{Cdc} 25 \mathrm{~A}$ and affects hepatic cystogenesis in a rat model of polycystic kidney disease. J Clin Invest 2008; 118: 3714-3724.

[26] Sun H, Li QW, Lv XY, Ai JZ, Yang QT, Duan JJ, Bian GH, Xiao Y, Wang YD, Zhang Z, Liu YH, Tan RZ, Yang Y, Wei YQ, Zhou Q. MicroRNA-17 post-transcriptionally regulates polycystic kidney disease-2 gene and promotes cell proliferation. Mol Biol Rep 2010; 37: 2951-2958.

[27] Petri A, Lindow M, Kauppinen S. MicroRNA silencing in primates: towards development of novel therapeutics. Cancer Res 2009; 69: 393-395.

[28] Wang G, Kwan BC, Lai FM, Chow KM, Li PK, Szeto CC. Elevated levels of miR-146a and miR-155 in kidney biopsy and urine from patients with IgA nephropathy. Dis Marker 2011; 30: 171-179.

[29] Wang G, Tam LS, Kwan BC, Li EK, Chow KM, Luk CC, Li PK, Szeto CC. Expression of miR-146a and miR-155 in the urinary sediment of systemic lupus erythematosus. Clin Rheumatol 2012; 31: 435-440.

[30] Kong AP, Kang X, Choi KC, Wang G, Chan MH, Ho CS, Chan I, Wong CK, Chan JC, Szeto CC. Associations between microRNA (miR-21, 126, 155 and 221), albuminuria and heavy metals in Hong Kong Chinese adolescents. Clinica Chimica Acta 2012; 413: 1053-1057. 


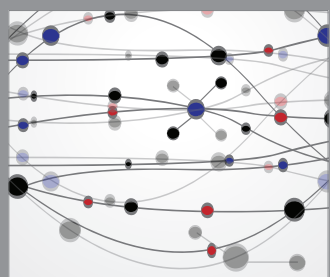

The Scientific World Journal
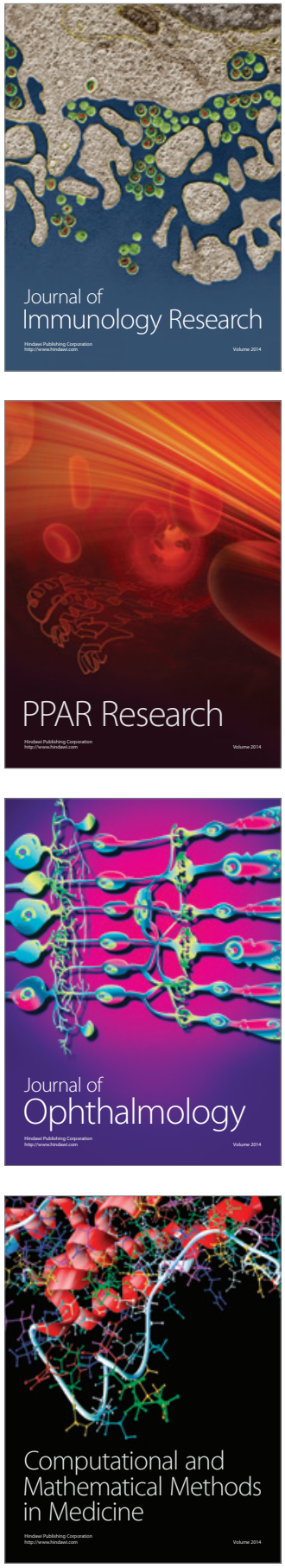

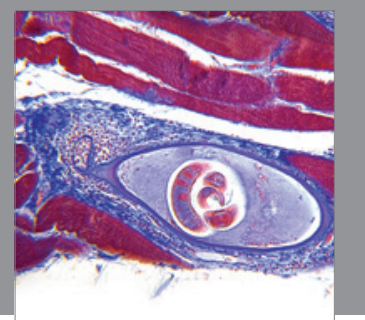

Gastroenterology

Research and Practice
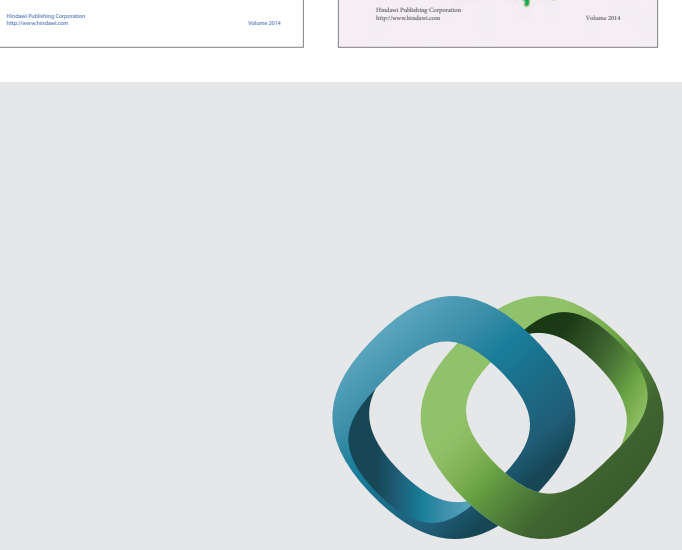

\section{Hindawi}

Submit your manuscripts at

http://www.hindawi.com
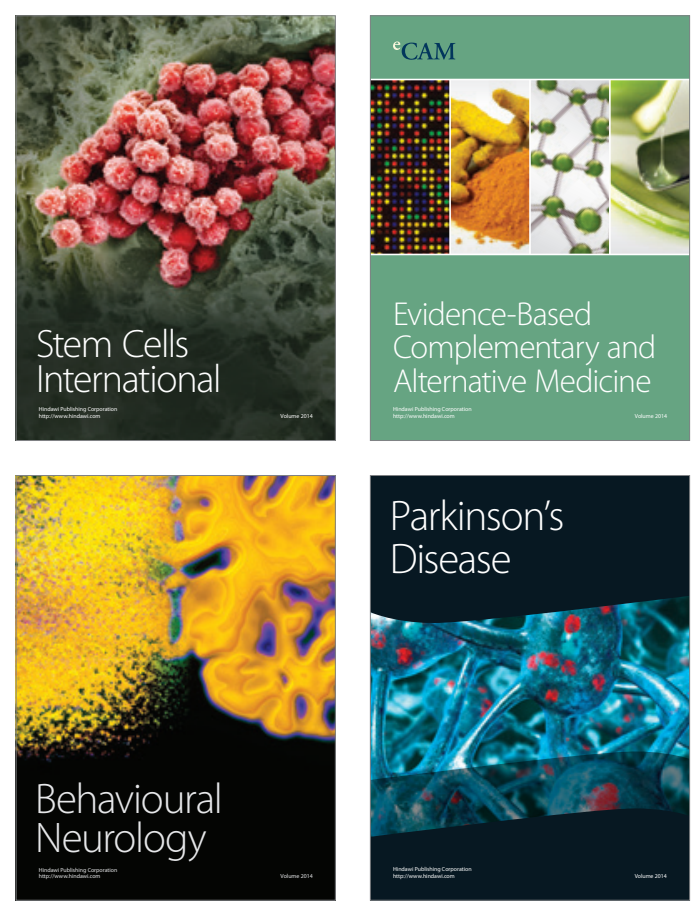

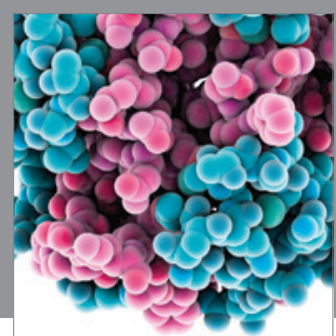

Journal of
Diabetes Research

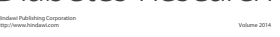

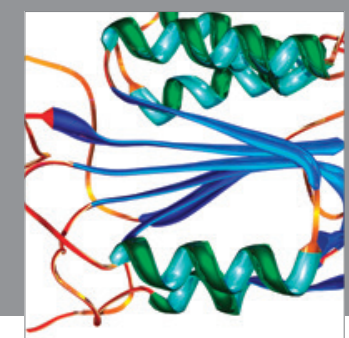

Disease Markers
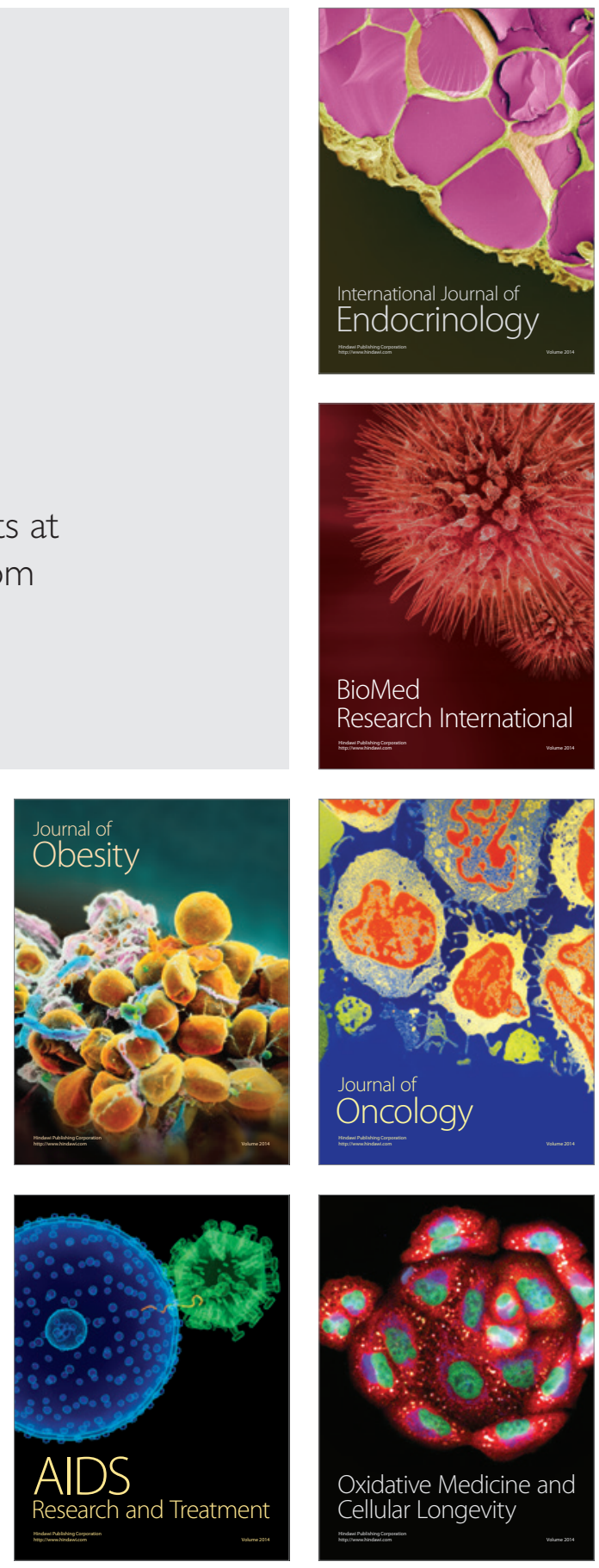